\title{
On a Certain Semilinear Parabolic System Related to the Lotka-Volterra Ecological Model
}

\author{
By \\ Masayasu MimURA* and Takaaki NisHIDA**
}

\begin{abstract}
A mathematical model proposed to explain the horizontal structure of prey and predator populations is represented by a semilinear parabolic system of equations. In this paper some mixed problems for this kind of system, Lotka-Volterra system, are considered and asymptotic behaviors of the solutions are investigated by use of Energy Method.

A remarkable fact is that, in opposition to Steele's conjecture (see [3]), the solution in the case of the Neumann boundary condition is asymptotically spatially homogeneous but does not tend to any constant steady state solution.
\end{abstract}

\section{§ 1. Introduction}

In 1930, a great mathematician, Vito Volterra published a book concerning the mathematical theory of the biological struggle for life [1]. His simplest model can be given in the form

$$
\begin{aligned}
& u_{t}=\left(\varepsilon_{1}-k v\right) u \\
& v_{t}=\left(-\varepsilon_{2}+k u\right) v . \quad(*)
\end{aligned}
$$

Here $u$ and $v$ are the population densities of the prey and the predator respectively, the positive constants $\varepsilon_{1}, \varepsilon_{2}$ and $k$ are the growth rate of $u$, the death rate of $v$ and the frequency of encounters. It is well known that the phase of (1-1) are closed with the center $u=\varepsilon_{2} / k$ and $v=\varepsilon_{1} / k$, if the initial data are positive.

Communicated by S. Matsuura, June 12, 1976.

* Department of Applied Mathematics, Konan University, Kobe 658, Japan.

** Department of Applied Mathematics and Physics, Kyoto University, Kyoto 606, Japan.

(*) This system was investigated by Lotka [2] in the theory of autocatalytic chemical reactions, so (1-1) is called the Lotka-Volterra model. 
Now it is interesting to consider (1-1) in the spatial inhomogeneity, because the majority of actual populations migrate in a given domain. If the flux of individuals is assumed to flow from higher densities to lower ones, then it is natural to introduce diffusion effects into (1-1). For a one dimensional spatial case, (1-1) may be written as

$$
\begin{aligned}
& u_{t}=d_{1} u_{x x}+\left(\varepsilon_{1}-k v\right) u \\
& u_{t}=d_{2} v_{x x}+\left(-\varepsilon_{2}+k u\right) v,
\end{aligned}
$$

where the diffusion coefficients $d_{1}$ and $d_{2}$ are both positive constants. Steele [3] proposed this system to explain the horizontal structure of prey and predator populations in a turbulent sea. In the sea, the phytoplankton and herbiovorous zooplankton are the prey and predator relationship. A main effect of plankton's movement is the current and turbulent lateral diffusion. However, Cassie [4] noted that plankton populations display spatially heterogeneity in spite of diffusion processes. These phenomena are called patchiness for planktons. From an ecological point of view, it is important to analyze the mechanics of patchiness effects [6]. Steele considered the initial-boundary value problem of (1-2) with zero flux boundary condition and conjectured that spatial inhomogeneites would appear, keeping the balance of the nonlinearity and diffusion effects. Hadeler, Heiden and Rothe [5] showed from thier numerical evidences that (1-2) had a non-trivial steady state solution with zero boundary condition. On the other hand, Murray [7] treated the same problem as Steele's under the same diffusion coefficients. Although his proof is not given in detail, he negatived Steele's conjecture.

This paper is motivated by Murray' paper and concerns with asymptotic behaviours of the solution of (1-2) under the appropriate initial-boundary conditions by using the well known ENERGY METHOD. The key of our result is the use of the conservation form derived from the Lotka-Volterra's model. 


\section{§ 2. Generalized Lotka-Volterra System with the Diffusion Effect.}

If $u_{i}(t, x) \quad(i=1,2, \ldots, n)$ denote the population densities of the interacting $i$-species, then the generalized system including (1-2) may be constructed as follows;

$$
u_{i_{t}}=d_{i} u_{i_{s s}}+\left(\varepsilon_{i}+\beta_{i}^{-1} \sum_{j=1}^{n} k_{i j} u_{j}\right) u_{i}
$$

for $i=1,2, \ldots, n$, where the diffusion coefficients $d_{i}$ are all positive constants, the constants $\varepsilon_{i}$ are the birth rates (if $\varepsilon_{i}>0$ ) or the death rates (if $\varepsilon_{i}<0$ ) of the $i$-species and $\left\{k_{i j}\right\}$ is an anti-symmetric constant matrix. If $k_{i j}>0$ (resp. $<0$ ), the $i$-species is a predator (resp. prey) to the $f$-species and $j$-species is a prey (resp. predator) to the $i$-species. Finally $\beta_{i}^{-1}$ are positive constants named "equivalence" numbers.

For the system (2-1), we consider some mixed problems with the domain $(t, x) \in(0,+\infty) \times(0, L)$. Here the initial conditions are given by

$$
u_{i}(0, x)=u_{i 0}(x), \quad x \in[0, L]
$$

and the boundary conditions by

$$
\begin{array}{ll}
u_{i}(t, 0)=h_{i}, u_{i}(t, L)=g_{i}, & t \geqq 0, \\
u_{i_{s}}(t, 0)=u_{i_{s}}(t, L)=0, & t \geqq 0,
\end{array}
$$

or

$$
\begin{aligned}
& u_{i}(t, 0)=u_{i}(t, L), \\
& u_{i_{s}}(t, 0)=u_{i_{s}}(t, L),
\end{aligned} \quad \mathrm{t} \geqq 0,
$$

where $h_{i}$ and $g_{i}$ are non-negative constant for $i=1,2, \ldots, n$. The conditions (2-3) and (2-4) mean a population reservoir and a barrier.

The local existence and the uniqueness of the solution of each mixed problem can be proved easily, so we do not write them down. 


\section{§ 3. Asymptotic Behaviours}

First of all we assume the following properties;

(1) (2-1) has at least one positive equilibrium state, that is, there exist positive constants $\left\{\bar{u}_{i}\right\}$ such that

$$
\beta_{i} \varepsilon_{i}+\sum_{j=1}^{n} k_{i j} \bar{u}_{j}=0 \quad(i=1,2, \ldots, n) .
$$

(2) the initial values $\left\{u_{i 0}(x)\right\}$ are positive and bounded in $x \in[0$, $L]$.

\section{3-1. Dirichlet Condition}

Let us consider the mixed problem (2-1), (2-2) and

$$
u_{i}(t, 0)=u_{i}(t, L)=\bar{u}_{i} .
$$

Now we introduce the following integral forms $E(t), E_{1}(t)$ and $E_{2}(t)$;

$$
\begin{aligned}
& E(U(t))=\sum_{i=1}^{n} \beta_{i} \int_{0}^{L}\left\{u_{i}-\bar{u}_{i}-\bar{u}_{i} \log \left(u_{i} / \bar{u}_{i}\right)\right\}(t, x) d x \\
& E_{1}(U(t))=\frac{1}{2} \sum_{i=1}^{n} \int_{0}^{L}\left(u_{i_{s}}\right)^{2}(t, x) d x
\end{aligned}
$$

and

$$
E_{2}(U(t))=\frac{1}{2} \sum_{i=1}^{n} \int_{0}^{L}\left(u_{i_{s x}}\right)^{2}(t, x) d x
$$

which play an important role in our discussions. We shall give some lemmas.

Lemma 3-1. Let $u_{i}(t, x)$ be a smooth solution of the mixed problem (2-1), (2-2) and (3-1). If we assume that $u_{i}(t, x) \leqq K \bar{u}_{i}$ for some positive constant $K$, then there exist positive constants $C_{1}(K)$ and $C_{2}(K)$ such that

$$
E(U(t))_{t}+C_{1}(K) E_{1}(U(t)) \leqq 0
$$

and 


$$
E_{1}(U(t))_{t}-C_{2}(K) E_{1}(U(t)) \leqq 0 .
$$

Proof. Differentiating (3-2) with respect to $t$, and using (3-1), we have

$$
\begin{aligned}
E(U(t))_{t} & =\sum_{i=1}^{n} \beta_{i} \int_{0}^{L}\left(1-\bar{u}_{i} / u_{i}\right) u_{i_{t}}(t, x) d x \\
& \left.=\sum_{i=1}^{n} \beta_{i} \int_{0}^{L}\left[d_{i}\left\{1-\bar{u}_{i} / u_{i}\right) u_{i_{x}}\right\}_{s}-d_{i} \bar{u}_{i}\left(u_{i_{s}} / u_{i}\right)^{2}\right](t, x) d x .
\end{aligned}
$$

Thus, if we put

$$
C_{1}(K)=\frac{2}{K^{2}} \min _{i}\left(\beta_{i} d_{i} / \bar{u}_{i}\right),
$$

then (3-5) can be obtained. In a similar manner, noting that $u_{i_{s s}}=0$ at $x=0$ and $x=L$ from (3-1), we have

$$
\begin{aligned}
E_{1}(U(t))_{t}= & \sum_{i=1}^{n} \int_{0}^{L}\left\{-d_{i}\left(u_{i_{s x}}\right)^{2}+\varepsilon_{i}\left(u_{i_{s}}\right)^{2}\right. \\
& \left.+\beta_{i}^{-1} \sum_{j=1}^{n} k_{i_{j}}\left(u_{j_{s}} u_{i}+u_{i_{s}} u_{j}\right) u_{i_{x}}\right\}(t, x) d x .
\end{aligned}
$$

Therefore, if $C_{2}(K)$ may be taken as $C_{2}(K)=2 \cdot \max _{i}\left(\left|\varepsilon_{i}\right|+2 \beta_{i}^{-1} \sum_{j=1}^{n}\left|k_{i j}\right|\right.$ $\left.\times K \bar{u}_{i}\right),(3-6)$ is obtained.

Lemma 3-2. In addition to the assumption of Lemma 3-1, if we assume $\bar{u}_{i} / K \leqq u_{i}(t, x)$ for $K \geqq 1$, then there exists a positive constant $C_{3}(K)$ such that

$$
E(U(t))-C_{3}(K) E_{1}(U(t)) \leqq 0 .
$$

Proof. Under the assumptions $\bar{u}_{i} K \geqq u_{i}(t, x) \geqq \bar{u}_{i} / K$, it holds that

$$
\bar{u}_{i}^{2}\left\{u_{i}-\bar{u}_{i}-\bar{u}_{i} \log \left(u_{i} / \bar{u}_{i}\right)\right\}^{2} \leqq K^{2} \int_{0}^{x}\left(u_{i}-\bar{u}_{i}\right)^{2} d x \int_{0}^{x}\left(u_{i_{s}}\right)^{2} d x
$$

and

$$
\left(u_{i}-\bar{u}_{i}\right)^{2} \leqq 2 K \bar{u}_{i}\left\{u_{i}-\bar{u}_{i}-\bar{u}_{i} \log \left(u_{i} / \bar{u}_{i}\right)\right\} .
$$

From the above inequalities, we obtain (3-10). Here it suffices to take $C_{3}(K)$ as $C_{3}(K)=4 K^{3} L^{2} \cdot \max _{i}\left(\beta_{i} / \bar{u}_{i}\right)$. 
Theorem 3-1. Consider the mixed problem (2-1), (2-2) and (3-1). Let the solution $u_{i}(t, x)$ be $\bar{u}_{i} / K \leqq u_{i}(t, x) \leqq K \bar{u}_{i}$ for some positive constant $K(\geqq 1)$. If

$$
E(U(0))=\sum_{i=1}^{n} \beta_{i} \int_{0}^{L}\left[u_{i 0}(x)-\bar{u}_{i}-\bar{u}_{i} \log \left\{u_{i 0}(x) / \bar{u}_{i}\right\}\right] d x<+\infty
$$

and

$$
E_{1}(U(0))=\frac{1}{2} \sum_{i=1}^{n} \int_{0}^{L}\left\{u_{i 0}(x)_{x}\right\}^{2} d x<+\infty
$$

then $u_{i}(t, x)$ approach $\bar{u}_{i}$ asymptotically with exponential order for $i=1,2, \ldots, n$.

Proof. Combining (3-5) and (3-6), we have

$$
\begin{gathered}
{\left[\left\{C_{2}(K)+1\right\} E(U(t))+C_{1}(K) E_{1}(U(t))\right]_{t}} \\
+C_{1}(K) E_{1}(U(t)) \leqq 0
\end{gathered}
$$

From (3-10) and (3-11), we obtain

$$
\begin{aligned}
& {\left[\left\{C_{2}(K)+1\right\} E(U(t))+C_{1}(K) E_{1}(U(t))\right]_{t}+C_{4}(K) \times} \\
& {\left[\left\{C_{2}(K)+1\right\} E(U(t))+C_{1}(K) E_{1}(U(t))\right] \leqq 0,}
\end{aligned}
$$

where $C_{4}(K)=C_{1}(K) /\left\{C_{3}(K) C_{2}(K)+C_{3}(K)+C_{1}(K)\right\}$. Thus (3-12) implies

$$
\begin{aligned}
\left\{C_{2}(K)\right. & +1\} E(U(t))+C_{1}(K) E_{1}(U(t)) \leqq e^{-C_{4}(K) t} \\
& \times\left[\left\{C_{2}(K)+1\right\} E(U(0))+C_{1}(K) E_{1}(U(0))\right],
\end{aligned}
$$

that is, $E(U(t))$ and $E_{1}(U(t))$ tend to zero as $t \rightarrow+\infty$. Consequently, $u_{i}(t, x)$ approach $\bar{u}_{i}$ asymptotically, because

$$
\left\{u_{i}(t, x)-\bar{u}_{i}\right\}^{2} \leqq 4 \sqrt{K \bar{u}_{i} E(U(t)) E_{1}(U(t))}
$$

hold for $i=1,2, \ldots, n$. Thus the proof is completed.

From the above discussions, we find that it is essential to get the uniformly boundedness of $u_{i}(t, x)$ such that $\bar{u}_{i} / K \leqq u_{i}(t, x) \leqq K \bar{u}_{i}$.

Lemma 3-3 (Murray). If the diffusion coefficients $d_{i}$ are all equal, that is, $d_{i}=d>0$, then the mixed problem (2-1), (2-2) and (3-1) 
has the a priori estimate $\bar{u}_{i} / K \leqq u_{i}(t, x) \leqq K \bar{u}_{i}$ for some positive constant $K$ which depends on the initial data.

Proof. We first define the functional $S(U)$ as

$$
S(U)=\sum_{i=1}^{n} \beta_{i}\left\{u_{i}-\bar{u}_{i}-\bar{u}_{i} \log \left(u_{i} / \bar{u}_{i}\right)\right\}
$$

If the solution $u_{i}(t, x)$ are substituted into (3-15), it follows that

$$
\begin{aligned}
S_{t} & =\sum_{i=1}^{n} \beta_{i}\left(1-\bar{u}_{i} / u_{i}\right) u_{i_{t}} \\
& =\sum_{i=1}^{n} \beta_{i}\left(1-\bar{u}_{i} / u_{i}\right)\left\{d u_{i_{s x}}+\left(\varepsilon_{i}+\beta_{i}^{-1} \sum_{j=1}^{n} k_{i}, u u_{j}\right) u_{i}\right\} \\
& =d \sum_{i=1}^{n} \beta_{i}\left\{u_{i}-\bar{u}_{i}-\bar{u}_{i} \log \left(u_{i} / \bar{u}_{i}\right)\right\}_{x x}-d \beta_{i} \sum_{i=1}^{n} \bar{u}_{i}\left(u_{i_{s}} / u_{i}\right)^{2} \\
& =d S_{x x}-d \beta_{i} \sum_{i=1}^{n} \bar{u}_{i}\left(u_{i_{s}} / u_{i}\right)^{2} .
\end{aligned}
$$

Therefore, we get the mixed problem with respect to $S$ as follows;

$$
S_{t}=d S_{x x}-z^{2}(t, x) \quad(t, x) \in(0,+\infty) \times(0, L),
$$

$$
\begin{gathered}
\left.S(0, x)=\sum_{i=1}^{n} \beta_{i}\left[u_{i 0}(x)-\bar{u}_{i}-\bar{u}_{i} \log \left\{u_{i 0}(x) / \bar{u}_{i}\right)\right\}\right], x \in[0, L], \\
S(t, 0)=S(t, L)=0, \quad t \geqq 0,
\end{gathered}
$$

where $z^{2}=\sum_{i=1}^{n} \beta_{i} \bar{u}_{i}\left(u_{i_{x}} / \bar{u}_{i}\right)^{2}$. By use of the well known Comparison theorem to the problem (3-17), we have

$$
0 \leqq S(t, x) \leqq \max _{x} \sum_{i=1}^{n} \beta_{i}\left[u_{i 0}(x)-\bar{u}_{i}-\bar{u}_{i} \log \left\{u_{\imath 0}(x) / \bar{u}_{i}\right\}\right]
$$

for $(t, x) \in(0,+\infty) \times(0, L)$. Thus we find that there exists a positive constant $K$ such that $\bar{u}_{i} / K \leqq u_{i}(t, x) \leqq K \bar{u}_{i}$ for $i=1,2, \ldots, n$.

Proposition 3-1. Let the diffusion coefficients $d_{\imath}$ be equal. If

$$
E(U(0))<+\infty \text { and } E_{1}(U(0))<+\infty \text {, }
$$

then the solution $u_{i}(t, x)$ approach to $\bar{u}_{i}$ asymptotically with exponential order for $i=1,2, \ldots, n$.

Proof. The proof follows from Theorem 3-1 and Lemma 3-3 directly, so we omit the details. 
In the general case that $d_{i}$ are different, we have no complete answer. At present our results are the followings;

Lemma 3-4. If $E(U(0))$ and $E_{1}(U(0))$ are sufficiently small, then there exists some positive constant $K$ such that $\bar{u}_{i} / K \leqq u_{i}(t, x)$ $\leqq K \bar{u}_{i}$ for $(t, x) \in(0,+\infty) \times(0, L)$.

Proof. Suppose that $\bar{u}_{i} / K \leqq u_{i}(t, x) \leqq K \bar{u}_{i}$, we have

$$
\begin{aligned}
\left\{u_{i}(t, x)-\bar{u}_{i}\right\}^{2} \leqq & \frac{4 K \bar{u}_{i} C_{1}(K)}{C_{2}(K)+1}\left\{\frac{C_{2}(K)+1}{C_{1}(K)} E(U(t))\right. \\
& \left.+E_{1}(U(t))\right\}=\bar{u}_{i}^{2} V_{K, E, E_{1}}^{2}(t)
\end{aligned}
$$

from (3-14). Hence, using (3-13), we obtain

$$
\left\{u_{i}(t, x)-\bar{u}_{i}\right\}^{2} \leqq \bar{u}_{i}^{2} V_{K, E, E_{1}}^{2}(0)
$$

for $(t, x) \in(0,+\infty) \times(0, L)$. Now, taking the values of $E(U(0))$ and $E_{1}(U(0))$ sufficiently small, we can get the number $K$ satisfying

$$
1+V_{K, E_{,} E_{1}}(0)<K \text { and } 1-V_{K, E, E_{1}}(0)>1 / \mathrm{K}
$$

where $V_{K, E_{1} E_{1}}(0)$ is positive. These conditions are consistent with $\bar{u}_{i} / K \leqq u_{i}(t, x) \leqq K \bar{u}_{\imath}$. Thus, the lemma is proved.

Proposition 3-2. Consider the mixed problem (2-1), (2-2) and (3-3). If $E(U(0))$ and $E_{1}(U(0))$ are sufficiently small, then $u_{i}(t, x)$ approach $\bar{u}_{i}$ asymptotically with exponential order for $i=1$, $2, \ldots, n$.

Proof. It is trivial to prove this proposition, so we omit it.

\section{3-2. Neumann Condition}

Let us consider the mixed problem (2-1), (2-2) and (2-4). The discussions in this part are almost the same as those of section 3-1. 
Lemma 3-5. Let $u_{i}(t, x)$ be a smooth solution of the mixed problem (2-1), (2-2) and (2-4) and satisfy $u_{i}(t, x) \leqq K \bar{u}_{i}$ for some positive constant $K$. If $E(U(0))<+\infty$ and $E_{1}(U(0))<+\infty$, it holds that

$$
\lim _{t \rightarrow \infty} E_{1}(U(t))=0 .
$$

Proof. In view of the boundary condition (2-4), (3-9) holds and gives

$$
E_{1}(U(t))_{t}+2 d E_{2}(U(t))-C_{2}(K) E_{1}(U(t)) \leqq 0
$$

and

$$
\left|E_{1}(U(t))_{t}\right|-2 \bar{d} E_{2}(U(t))-C_{2}(K) E_{1}(U(t)) \leqq 0,
$$

where $\underline{d}=\min \left(d_{1}, d_{2}, \ldots, d_{n}\right)$ and $\bar{d}=\max \left(d_{1}, d_{2}, \ldots, d_{n}\right)$. From (3-5) and (3-19), we obtain

$$
\left\{E_{1}(U(t))+\frac{C_{2}(K)}{C_{1}(K)} E(U(t))\right\}_{t}+2 d E_{2}(U(t)) \leqq 0 .
$$

Hence, we have

$$
\int_{0}^{t} E_{2}(U(\tau)) d \tau \leqq \frac{1}{2 d}\left\{\frac{C_{2}(K)}{C_{1}(K)} E(U(0))+E_{1}(U(0))\right\}
$$

Consequently, (3-20) and (3-22) give

$$
\int_{0}^{t}\left|E_{1}(U(\tau))_{\tau}\right| d \tau \leqq \frac{C_{2}(K)}{C_{1}(K)}\left(\frac{\bar{d}}{\underline{d}}+1\right) E(U(0))+\frac{\bar{d}}{\underline{d}} E_{1}(U(0)) .
$$

Here $\int_{0}^{t} E_{1}(U(\tau)) d \tau \leqq \frac{E(U(0))}{C_{1}(K)}$ is used. Then we have

$$
\lim _{t \rightarrow \infty} E_{1}(U(t))=0 \text {, }
$$

which completes the proof.

If $L$ is small, we can represent Lemma 3-5 more precisely,

Proposition 3-3. Under the assumption of Lemma 3-5, if $C_{2}(K)$ $<\frac{4 d}{L^{2}}$, then $E_{1}(U(t))$ tends to zero with exponential order as $t \rightarrow \infty$.

Proof. Noting the Poincaré's inequality 


$$
E_{1}(U(t)) \leqq \frac{L^{2}}{2} E_{2}(U(t)),
$$

we have from (3-19)

$$
E_{1}(U(t))_{t}+\left\{\frac{4 \underline{d}}{L^{2}}-C_{2}(K)\right\} E_{1}(U(t)) \leqq 0,
$$

which gives the proof directly.

Lemma 3-6. If $E(U(0))$ and $E_{1}(U(0))$ are sufficiently small, there exists some positive constant $K$ such that $\bar{u}_{i} / K \leqq u_{i}(t, x) \leqq K \bar{u}_{i}$ for $(t, x) \in(0,+\infty) \times(0, L)$.

Proof. For any fixed number $s \in[0, L]$, we have

$$
\begin{aligned}
\left\{u_{i}(t, s)\right. & \left.-\bar{u}_{i}\right\}^{2}-\left\{u_{i}(t, x)-\bar{u}\right\}^{2} \\
& =2 \int_{x}^{s}\left\{u_{i}(t, \xi)-\bar{u}_{i}\right\} u_{i_{s}}(t, \xi) d \xi .
\end{aligned}
$$

Supposing that $\bar{u}_{i} K \leqq u_{i}(t, x) \leqq K \bar{u}_{i}$, then (3-26) gives

$$
\left\{u_{i}(t, s)-\bar{u}_{i}\right\}^{2} \leqq 4 \sqrt{K \bar{u}_{i} E(U)} \overline{(t))} \overline{E_{1}}\left(\overline{U(t))}+\left\{u_{i}(t, x)-\bar{u}_{i}\right\}^{2} .\right.
$$

Integrating (3-27) from 0 to $L$ with respect to $x$, we get

$$
\left\{u_{i}(t, s)-\bar{u}_{i}\right\}^{2} \leqq 4 \sqrt{K \bar{u}_{i} E(U(t)) E_{1}(U(t))}+2 K \bar{u}_{i} E(U(t)) / L .
$$

Choosing $E(U(0))$ and $E_{1}(U(0))$ sufficiently small, we find that $u_{i}(t, x)$ are consistent with $\bar{u}_{i} / K \leqq u_{i}(t, x) \leqq K \bar{u}_{i}$, by using the procedure analogous to that of Lemma 3-4. Therefore the proof is given.

Theorem 3-2. If $E(U(0))$ and $E_{1}(U(0))$ are sufficiently small, then the solution $u_{i}(t, x)$ of the mixed problem (2-1), (2-2) and (2-4) become to be spatial homogeneous asymptotically and

$$
\lim _{t \rightarrow \infty}\left(u_{1}(t, x), u_{2}(t, x), \ldots, u_{n}(t, x)\right) \in\left\{U \mid S(U)=\frac{E(\infty)}{L}\right\} .
$$

Proof. For some fixed number $p \in[0, L]$, it follows that 


$$
\begin{aligned}
\left\{u_{i}(t, x)-u_{i}(t, p)\right\}^{2} & \leqq 2 \int_{p}^{x}\left\{u_{i}(t, \xi)-u_{i}(t, p)\right\} u_{i_{s}}(t, \xi) d \xi \\
& \leqq 4 K \bar{u}_{i} \sqrt{L E_{1}(U(t))} .
\end{aligned}
$$

Hence, by using Lemma $3-5$, we find that $u_{i}(t, x)$ become to be spatially homogeneous for $i=1,2, \ldots, n$, as $t \rightarrow+\infty$. On the other hand, $S(U(t, x))$ is continuous in $x$, so there exists some number $x_{0}(t)$ depending on $t$, such that

$$
S\left(U\left(t, x_{0}(t)\right)\right)=\frac{1}{L} \int_{0}^{L} S(U(t, \xi)) d \xi=\frac{E(U(t))}{L} .
$$

Thus, it follows that

$$
S(U(t, x))-\frac{E(U(t))}{L}=\sum_{i=1}^{n} \beta_{i} \int_{x_{0}}^{x}\left(1-\bar{u}_{i} / u_{i}\right) u_{i_{s}}(t, \xi) d \xi
$$

and that

$$
\left|S(U(t, x))-\frac{E(U(t))}{L}\right| \leqq(1+K)\left(\sum_{i=1}^{n} \beta_{i}^{2}\right)^{\frac{1}{2}} \sqrt{L E_{1}(U(t))}
$$

which implies the proof.

Proposition 3-4. Let the diffusion coefficients $d_{i}$ be all equal. If $E(U(0))<+\infty$ and $E_{1}(U(0))<+\infty$, then the solution $u_{i}(t, x)$ has the same property as that of Theorem 3-2.

Proof. The proof is obvious, so we omit it.

From Theorem 3-2 and Proposition 3-4, we can get the following remark;

Remark 3-2. Let the solution $u_{i}(t, x)$ satisfy $\bar{u}_{i} / K \leqq u_{i}(t, x) \leqq K \bar{u}_{i}$. If $E(U(0))<+\infty$ and $E_{1}(U(0))<+\infty$, the necessary and sufficient condition of $\lim _{t \rightarrow \infty} u_{i}(t, x)=\bar{u}_{i}$ is $E(U(\infty))=0$.

Proposition 3-5. Let $u_{i}(t, x)$ satisfy $\bar{u}_{i} / K \leqq u_{i}(t, x) \leqq K \bar{u}_{i}$ for some positive constant $K$. If $E(U(0)), E_{1}(U(0)), \bar{u}_{i}$ and $d_{i}$ are so chosen as 


$$
E(U(0))>\frac{L^{2} C_{4}(K)}{4 \underline{d}-L^{2} C_{2}(K)} E_{1}(U(0)) \text { and } 4 \underline{d}>L^{2} C_{2}(K),
$$

where $C_{4}(K)=2 K^{2} \max _{i}\left(\beta_{i} d_{i} / \bar{u}_{i}\right)$, then it follows $E(U(\infty))>0$.

Proof. From (3-25), we get

$$
\left\{\frac{4 \underline{d}}{L^{2}}-C_{2}(K)\right\} \int_{0}^{t} E_{1}\left(U(\tau) d \tau \leqq E_{1}(U(0)) .\right.
$$

Combining (3-33) and $E(U(t))_{t} \geqq-C_{4}(K) E_{1}(U(t))$, we have

$$
E(U(t)) \geqq E(U(0))-\frac{C_{4}(K)}{\frac{4 d}{L^{2}}-C_{2}(K)} E_{1}(U(0)) .
$$

Here $\frac{4 \underline{d}}{L^{2}}-C_{2}(K)>0$ is used. Thus (3-34) gives the proof directly.

\section{§4. Concluding Remarks}

By applying arguments similar to those used in this paper, we can treat the asymptotic problem in the case of the periodic boundary condition (2-5).

We could not argue about the Dirichlet boundary condition (2-3) deeply. When the non-trivial solution $w_{i}(x)$ of the boundary value problem (2-1) and (2-3) exist in the neighbourhood of $\bar{u}_{i}$, we can prove the following;

Theorem 4-1. Instead of the assumptions of $\left\{K_{i j}\right\}$, let us assume that $\left\{\beta_{i}^{-1} \bar{u}_{i} k_{i j}\right\}$ is an anti-symmetric matrix. ${ }^{(*)}$ Assume that there exist $w_{i}(x)$ satisfying

$$
\begin{aligned}
& d_{i} w_{i s s}+\left(\varepsilon_{i}+\beta_{i}^{-1} \sum_{j=1}^{n} k_{i j} w_{j}\right) w_{i}=0, \quad x \in(0, L) \\
& w_{i}(0)=h_{i}, \quad u_{i}(L)=g_{i}
\end{aligned}
$$

such that

$$
\left|w_{i}(x)-\bar{u}_{i}\right| \leqq M \text { for } x \in[0, L] \text { and } 4 \underline{d}-L^{2} C_{5}(M)>0,
$$

(*) If $k_{i j}$ is sufficiently small, we can remove this condition. 
where $C_{5}(M)=4 \max _{i, j}\left(\beta_{i}^{-1}\left|k_{i j}\right|\right) M n . \quad$ If $E_{0}(V(0))=\frac{1}{2} \sum_{i=1}^{n} \int_{0}^{L}\left\{u_{i 0}(x)-\right.$ $\left.w_{i}(x)\right\}^{2} d x$ and $E_{1}(V(0))$ are sufficiently small according to $M$, the solution $u_{i}(t, x)$ of the mixed problem (2-1), (2-2) and (2-3) tend to $w_{i}(x)$ with exponential order for $i=1,2, \ldots, n$.

Proof. Substituting $v_{i}(t, x)=u_{i}(t, x)-w_{i}(x)$ into (2-1), we get the following system with respect to $v_{i}$;

$$
v_{i_{i}}=d_{i} v_{i_{s s}}+\left(\varepsilon_{i}+\beta_{i}^{-1} \sum_{j=1}^{n} k_{i j} u_{j}\right) v_{i}+\beta_{i}^{-1} \sum_{j=1}^{n} k_{i j} w_{i} v_{j} .
$$

Here the initial conditions are given by

$$
v_{i}(0, x)=u_{i 0}(x)-w_{i}(x) \quad x \in[0, L]
$$

and the boundary conditions by

$$
v_{i}(t, 0)=v_{i}(t, L)=0 \quad t \geqq 0 .
$$

Supposing that $\left|u_{i}(t, x)-\bar{u}_{i}\right| \leqq M$ for $(t, x) \in(0,+\infty) \times[0, L]$, we get

$$
E_{0}(V(t))_{t} \leqq-2 d E_{1}(V(t))+C_{5}(M) E_{0}(V(t))
$$

Let $M$ choose as $4 \underline{d}-L^{2} C_{5}(M)>0$. Then, by use of Poincaré's inequality, $E_{0}(V(t))$ can be estimated by

$$
E_{0}(V(t)) \leqq e^{-s t} E_{0}(V(0))
$$

for some positive constant $s$. By using the same argument as $E_{0}$, we have

$$
\begin{aligned}
E_{1}(V(t))_{t} \leqq & -2 d E_{2}(V(t))+\frac{C_{5}(M)}{2}\left(\frac{\left|W_{x}\right|}{M}+3\right) E_{1}(V(t)) \\
& +\frac{C_{5}(M)\left|W_{x}\right|}{2 M} E_{0}(V(t))
\end{aligned}
$$

where $\left|W_{x}\right|=\max _{i}\left(\max _{x}\left|w_{i_{s}}(x)\right|\right)$. From (4-4) and (4-6), it follows

$$
\begin{gathered}
\left.\left\{E_{0}(V(t))+h E_{1}(V(t))\right\}_{t} \leqq-\left\{2 d-\frac{C_{5}(M) h}{2}\left(\frac{\left|W_{x}\right|}{M}\right)+3\right)\right\} \\
\times E_{1}(V(t))+C_{5}(M)\left(1+\frac{h}{2 M}\left|W_{x}\right|\right) E_{0}(V(t)) .
\end{gathered}
$$

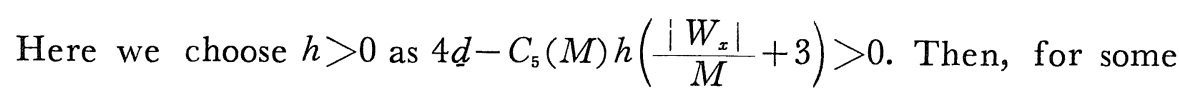


$m>0,(4-7)$ can be rewritten as

$$
\begin{aligned}
\left\{E_{0}(V(t))\right. & \left.+h E_{1}(V(t))\right\}_{t} \leqq-\frac{m}{h}\left\{E_{0}(V(t))+h E_{1}(V(t))\right\} \\
& +p E_{0}(V(t))
\end{aligned}
$$

where $p=\frac{m}{h}+C_{5}(M)\left(1+\frac{h}{2 M}\left|W_{x}\right|\right)$. Applying (4-5) to (4-8), $E_{1}$ can be estimated as follows;

$$
\begin{aligned}
E_{0}(V(t)) & +h E_{1}(V(t)) \leqq \frac{p E_{0}(V(0))}{\frac{m}{h}-s} e^{-s t}+\left\{E_{0}(V(0))\right. \\
& \left.+h E_{1}(V(0))+\frac{p E_{0}(V(0))}{s-\frac{m}{h}}\right\} e^{-\frac{m}{h} t}, \quad\left(\frac{m}{h} \neq s\right)
\end{aligned}
$$

or

$$
\leqq\left\{(p t+1) E_{0}(V(0))+h E_{1}(V(0))\right\} e^{-s t} \quad\left(\frac{m}{h}=s\right)
$$

Hence, if $E_{0}(V(0))$ and $E_{1}(V(0))$ are choosen as sufficiently small, $v_{i}(t, x)$ tend to zero with exponential order, because of

$$
v_{i}^{2}(t, x) \leqq 2 \sqrt{E_{0}(V(t)) E_{1}(V(t))} .
$$

Thus, the proof is completed.

\section{Acknowledgements}

The authors would like to thank Professor M. Yamaguti, Kyoto University for many discussions and suggestions in this work. It is also noted with sincere appreciation that the referee extended his help to offer many suggestions.

\section{References}

[1] Volterra, V., Lécons sur la Théorie Mathématique de la lutte pour la vie, GauthierVillars, Paris. (1931).

[2] Lotka, J. A., P roc. Natn. Acad. Sci., U. S. 6 (1920) 410-415.

[3] Steele, J.H., Natu re, Lond., 248 (1974) 83.

[4] Cassie, R. M., Oceanrg. Mar. Biol. Ann Rev., 1 (1963) 223-252.

[5] Hader, K. P., an der Heiden, U. and Rothe, F., J. Math. Biology, 1 (1974) 165-176.

[6] Dubois, D. M., Ecological Modelling, 1 (1974) 67-80.

[7] Murray, J.D., J. Theor. Biology, 52 (1975) 459-469. 